\title{
Optimized calibration of cryogenic silicon thermodiodes
}

\author{
O.M. Ivashchenko', Yu.M. Shwarts ${ }^{2}$, M.M. Shwarts ${ }^{3}$, D.P. Kopko ${ }^{4}$, M.I. Sypko \\ V. Lashkaryov Institute of Semiconductor Physics, National Academy of Sciences of Ukraine, \\ 41, prosp. Nauki, 03028 Kyiv, Ukraine \\ Phone/ Fax: +38/044/525-7463 \\ ${ }^{1}$ E-mail: iva@isp.kiev.ua \\ ${ }^{2}$ E-mail: shwarts@isp.kiev.ua \\ ${ }^{3}$ E-mail: mshwarts@isp.kiev.ua \\ ${ }^{4}$ E-mail:kdp2007@ukr.net
}

\begin{abstract}
Shown in this paper is the efficiency of smoothing cubic spline approximation aimed at optimization of calibration of cryogenic silicon diode thermometers (SDTs). The proposed algorithm allows to significantly reduce time and material costs associated with calibration of SDTs.
\end{abstract}

Keywords: spline, free-knot, silicon diode, temperature sensor, calibration.

Manuscript received 21.12.10; revised manuscript received 10.08.11; accepted for publication 14.09.11; published online 30.11.11.

\section{Introduction}

The procedure aimed at calibration of temperature sensors includes stabilization and measurement of a selected temperature, maintaining a stable operation current, measurement of electrical signals (voltages, currents) and resistances. To achieve high precision in measurements, it is necessary to use highly accurate instruments based on modern microcontrollers as well as automated systems to control and keep temperature. Thus, calibration is a complex technical task that requires significant time and material costs, which substantially increases the cost of thermal sensors.

To solve this task, proposed in the paper [1] is the method that simplifies calibration of interchangeable cryogenic thermistors of the Cernox type within several Kelvins by reducing the number of measurements from 50 down to 30 within the temperature range $1.6-300 \mathrm{~K}$. The method is based on the heuristic analysis of large array experimental data. Algorithm proposed by the authors using the model of a cubic spline with free knots allows finding the position and the minimum number of experimental points within the operation temperature range for thermistors necessary for calculation of their thermometric characteristics (TCh). At the same time, the algorithm requires a number of specific criteria, for example, the fourth derivative of the sensor TCh should not be equal to zero.
Previously, we reported on the development of cryogenic silicon diode thermometers (SDTs) [2]. As shown in [3], their individual TCh are well described by a cubic spline with free knots, when using a large amount of experimental data. At the same time, the method [1] can not be used to optimize the thermal diode calibration due to violations of the criterion for its applicability: a weak temperature dependence of the sensitivity for these SDTs within the range 100 to 373 $\mathrm{K}$ does not allow determining its derivatives with a high precision.

The purpose of this article is to develop an optimization algorithm aimed at calibration of interchangeable silicon diode thermometers by minimizing the number of measurements necessary to calculate TCh with the required accuracy by using the model of smoothing cubic spline with free knots.

\section{Samples and experimental technique}

To develop a method for optimizing the calibration of the developed SDTs, we made 300 samples. Before grading the SDTs, we exposed them up to 30 thermal shocks (room temperature - liquid nitrogen and room temperature - liquid helium), which was followed by controlling the stability of sensor readings at temperatures of liquid nitrogen and/or helium. Calibration of the SDTs for three values of the operation 
current $I$ was carried out using the automated metrological stand UGT-A with a flow cryostat [2]. According to our estimates, the total measurement error did not exceed $\pm 40 \mathrm{mK}$, the standard deviation (SD) of the random component of the experimental error in the temperature equivalent was less than $10 \mathrm{mK}$.

\section{Results}

The detailed calibration of the sensor volume sample $\mathrm{C}$ was carried out using a large number of experimental points (for $I=1 \mu \mathrm{A}$, the number of experimental points $N$ $=169$ within the range 4.2 to $373 \mathrm{~K}$, and for $I=10$ and $100 \mu \mathrm{A} \mathrm{N}=106$ within the range 30 to $373 \mathrm{~K}$ ). According to the criteria selected by us in [3], using a cubic smoothing spline found were the approximating functions for experimental dependences $U_{i}(T)$ as well as temperature sensitivity of sensors $S_{i}=d F_{i} / d T$. Moreover, as shown in Table 1 , to calculate $F_{i}(T)$ for similar SDTs we used splines with the same number and distribution of knots over the temperature axis.

Table 1. Parameters of the approximating spline $F_{i}(T)$ for SDTs

\begin{tabular}{|l|l|l|l|l|l|l|}
\hline \multirow{2}{*}{$\begin{array}{l}\text { Cur- } \\
\text { rent, } \\
\mu \mathrm{A}\end{array}$} & $\begin{array}{l}\text { SDT } \\
\text { num- } \\
\text { ber }\end{array}$ & \multicolumn{4}{|c|}{$\begin{array}{l}\text { Amounts of non-repeated knots } \\
\text { in temperature subranges, }\end{array}$} & $\begin{array}{l}\text { Total } \\
\text { amount } \\
\text { of } \\
\text { knots }\end{array}$ \\
\hline \multirow{4}{*}{1} & $1.2 \ldots 20$ & $20 \ldots 50$ & $50 \ldots 150$ & $150 \ldots 373$ & \\
\cline { 2 - 7 } & 2 & 12 & 6 & 8 & 6 & 32 \\
\cline { 2 - 7 } & 3 & 12 & 6 & 7 & 5 & 30 \\
\hline \multirow{3}{*}{10} & 1 & & 5 & 8 & 5 & 31 \\
\cline { 2 - 7 } & 2 & & 5 & 10 & 5 & 20 \\
\cline { 2 - 7 } & 3 & & 5 & 7 & 5 & 17 \\
\hline \multirow{3}{*}{100} & 1 & & 6 & 8 & 5 & 19 \\
\cline { 2 - 7 } & 2 & & 6 & 8 & 5 & 19 \\
\cline { 2 - 7 } & 3 & & 7 & 7 & 5 & 19 \\
\hline
\end{tabular}

To determine the level of interchangeability for the developed SDTs, first we found the standard characteristics $\bar{F}(T)$ for each operation current:

$\bar{F}(T)=\frac{\sum_{i=1}^{C} F_{i}(T)}{C}$,

where $\mathrm{C}=26$ for $I=1 \mu \mathrm{A}$ and $\mathrm{C}=11$ for currents 10 and $100 \mu \mathrm{A}$, and then the temperature deviations of individual calculated TChs from the standard

$\Delta_{F_{i}}(T)=\frac{F_{i}(T)-\bar{F}(T)}{d \bar{F}(T) / d T}$.

The performed analysis has shown that the developed sensors can be characterized by five tolerance bands (Table 2):
Table 2. Tolerance bands for interchangeability of the developed SDTs

\begin{tabular}{|c|l|l|l|}
\hline $\begin{array}{c}\text { Ope- } \\
\text { ration } \\
\text { cur- } \\
\text { rent, } \\
\mu \mathrm{A}\end{array}$ & $\begin{array}{c}\text { Designa- } \\
\text { tion of } \\
\text { tolerance } \\
\text { bands }\end{array}$ & $\begin{array}{c}\text { Deviation from } \\
\text { the standard } \\
\text { TCh } \\
\text { (in temperature } \\
\text { equivalents), K }\end{array}$ & \multicolumn{1}{|c|}{$\begin{array}{c}\text { Temperature } \\
\text { range, } \mathrm{K}\end{array}$} \\
\hline 1 & $\mathrm{~A}$ & \pm 0.25 & $4.2-373$ \\
\cline { 2 - 4 } & $\mathrm{B}$ & \pm 0.35 & $4.2-50$ \\
& \pm 0.25 & $50-373$ \\
\cline { 2 - 4 } & $\mathrm{C}$ & \pm 0.60 & $4.2-373$ \\
\cline { 2 - 4 } & $\mathrm{D}$ & \pm 0.50 & $4.2-100$ \\
& $\mathrm{E}$ & \pm 2.5 & $100-373$ \\
\hline \multirow{5}{*}{10} & $\mathrm{~A}$ & \pm 0.35 & $4.2-373$ \\
\cline { 2 - 4 } & $\mathrm{B}$ & \pm 0.50 & $30-373$ \\
\cline { 2 - 4 } & $\mathrm{C}$ & \pm 1 & $30-373$ \\
\cline { 2 - 4 } & $\mathrm{D}$ & \pm 1.5 & $30-373$ \\
\cline { 2 - 4 } & $\mathrm{E}$ & \pm 3 & $30-373$ \\
\hline 100 & $\mathrm{~A}$ & \pm 0.35 & $30-373$ \\
\cline { 2 - 4 } & $\mathrm{B}$ & \pm 0.50 & $30-373$ \\
\cline { 2 - 4 } & $\mathrm{C}$ & \pm 0.50 & $30-373$ \\
\hline & $\mathrm{D}$ & \pm 1.50 & $78-373$ \\
\hline & $\mathrm{E}$ & \pm 3 & $30-373$ \\
\hline
\end{tabular}

The deviation $\Delta_{F_{i}}(T)$ of the SDT group from the band $\mathrm{C}$ for the current $1 \mu \mathrm{A}$ is illustrated in Fig 1 . One can see that the maximum deviation value over the whole operation range for these SDTs does not exceed $\pm 0.6 \mathrm{~K}$.

As seen from the data adduced in Table 2, the sensors of the A band are the closest ones to the standard over the whole operation temperature range.

In general, $\Delta_{F_{i}}(T)$ for all operation currents does not exceed $\pm 3 \mathrm{~K}$ (band $\mathrm{E}$ ). The data obtained on the interchangeability of the SDTs and the ability to describe the experimental TChs by splines with the same parameters show that the algorithm of optimized calibration can be applied to all the developed series of SDTs.

Taking $\bar{F}(T)$ as a model function, we find the minimum number of experimental points $M<N$ and their location within the operation temperature range $\left[\mathrm{T}_{1}\right.$, $\mathrm{T}_{\mathrm{M}}$ ] for chosing such a function $\bar{F}^{\prime}(T)$ for which the condition

$$
\max _{T \in\left[T_{1}, T_{M}\right]}(|\Delta(T)|) \leq 0.1 \cdot \Delta_{\text {exp }},
$$

where

$\Delta(T)=\frac{\bar{F}^{\prime}(T)-\bar{F}(T)}{d \bar{F} / d T}$ 


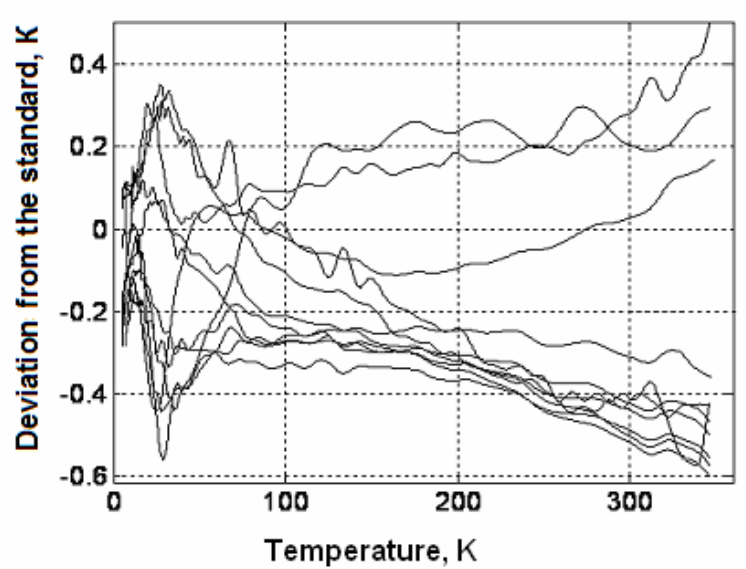

Fig. 1. Deviations of individual calculated TChs for groups of the SDTs from the standard curve. Operation current is $1 \mu \mathrm{A}$. The data are presented for samples from the band C.

From the mathematical viewpoint, the desired function of the spline can be represented as:

$$
\bar{F}^{\prime}(T)=\left.\sum_{j=1}^{n}\right|_{j} B_{j}(T)
$$

where ${ }_{i} b_{j}$ are the coefficients of the spline, $B_{j}(T)-\beta-$ spline of the third degree computed for the $j$-th part between $n$-knots.

To define $\bar{F}^{\prime}(T)$, let us take the vector of knot approximation as ${ }_{1} \mathrm{y}=\left\{1 \mathrm{y}_{j} ; j=1 \ldots n\right\}$ and the data table $\left\{T_{\mathrm{i}}, U_{\mathrm{i}} ; i=1 \ldots M\right\}$. The voltage values corresponding to the temperature $T_{i}$ are found from the model function $\bar{F}(T)$, i.e. $U_{i}=\bar{F}_{i}(T)$. At the first stage of searching, we find the knots allowing for description of all the features inherent to the function $\bar{F}(T)$. At the same time, to limit the search range we should use the temperature points $M_{\text {int }}=n-4$ (the case of interpolation) coinciding with the knots (Fig. 2).

As a result of interpolation, we have obtained the temperature intervals with their corresponding errors. From this series of errors $\sigma$, we find the $k$-th and $l$-th intervals with the highest $\sigma_{k}$ and lowest $\sigma_{l}$ errors, respectively. According to [4], reduction of the mesh size for interpolation significantly reduces the errors of cubic spline interpolation. Let us make a decrease in the $k$-th and the increase in $l$-th intervals by the value $\delta T$ up to the moment when for a certain set of knots in all the mesh intervals the errors become practically the same, that is, until the condition

$\sigma_{1} \approx \sigma_{2} \approx \ldots \approx \sigma_{M-1}$

will be fulfilled.

This criterion allows us to find the optimal mesh of knots $\tau_{o p t}$, using which we significantly reduce the maximum interpolation error. However, the use of knots $\tau_{\text {opt }}$ to approximate the actual experimental data does not provide the condition (3). Therefore, it is necessary to apply a smoothing spline constructed by the least squares method, which requires additional experimental points located between the knotes in the temperature scale (Fig. 3).

As result of simulating the function $\bar{F}(T)$ by the function $\bar{F}^{\prime}(T)$ with account of the condition (3), were found the optimum temperature meshes $T_{\text {opt }}$ and knots of approximation $\tau_{\text {opt }}$ for calibration at three operation currents. Their numbers were for the operation currents as follows: for $1 \mu \mathrm{A} \quad T_{o p t}=95$ points, $\tau_{\text {opt }}=42 \mathrm{knots}$; for $10 \mu \mathrm{A} T_{\text {opt }}=31$ points, $\tau_{\text {opt }}=18 \mathrm{knots}$; for $100 \mu \mathrm{A} T_{\text {opt }}=$ 36 points, $\tau_{\text {opt }}=23$ knots.

The found in this way optimal meshes $T_{o p t}$ and $\tau_{o p t}$ were used to simulate $F_{i}(T)$ and $S_{i}(T)$ for the investigated SDTs under various operation currents. The obtained results have been illustrated in Figs 4 to 6 . Regardless of SDT affiliation to any tolerance band, using the same meshes $T_{o p t}$ and $\tau_{o p t}$ allows to approximate $F_{i}(T)$ with the accuracy not worse than $4 \mathrm{mK}$.

Fig. 2. Arrangement of temperature points $T_{i}$ and knots of the interpolation spline $\tau_{j}$ on the temperature axis $\left[T_{1}, T_{\text {Mint }}\right]$. The operation current of the sensor is $1 \mu \mathrm{A}$.

Fig. 3. Arrangement of experimental points $T_{i}$ and knots of the approximating spline $\tau_{j}$ on the temperature axis $\left[T_{1}, T_{\text {Mapp }}\right]$. The operation current of the sensor is $1 \mu \mathrm{A}$.

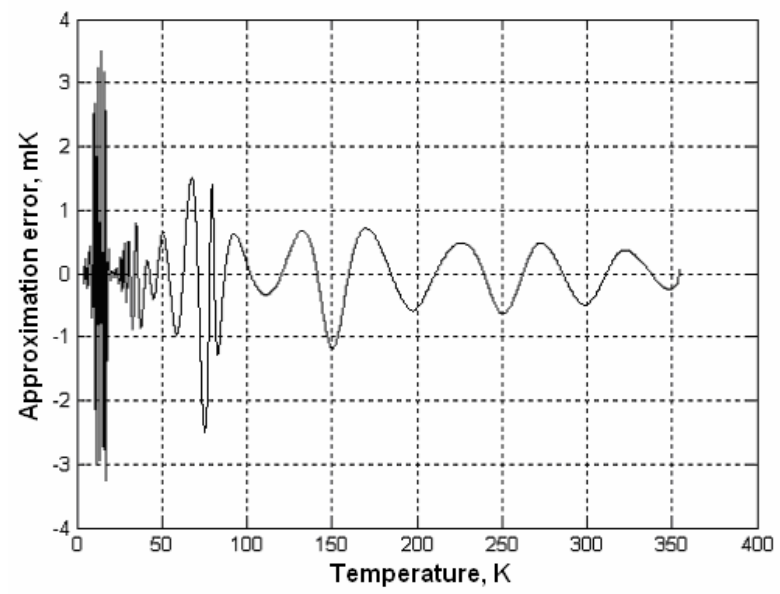

Fig. 4. Approximation error for the function $F_{i}(T)$ describing the sensor NKGPI20 when using the optimal meshes $T_{\text {opt }}$ and $\tau_{\text {opt }}$. The operation current of the sensor is $1 \mu \mathrm{A}$.

\section{(C) 2011, V. Lashkaryov Institute of Semiconductor Physics, National Academy of Sciences of Ukraine}




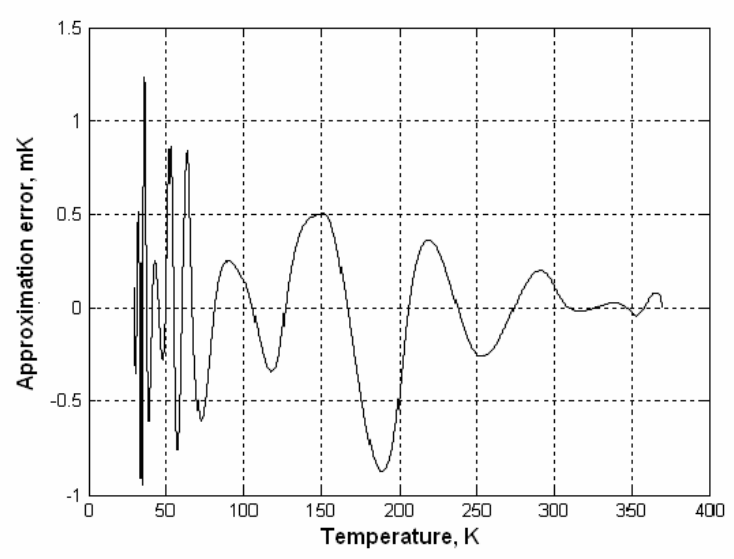

Fig. 5. Approximation error for the function $F_{i}(T)$ describing the sensor 220 when using the optimal meshes $T_{\text {opt }}$ and $\tau_{\text {opt }}$. The operation current of the sensor is $10 \mu \mathrm{A}$.

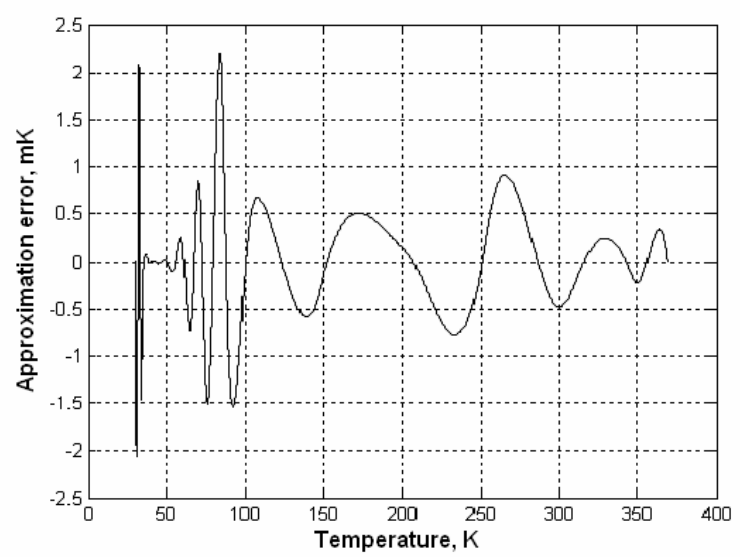

Fig. 5. Approximation error for the function $F_{i}(T)$ describing the sensor 233 when using the optimal meshes $T_{\text {opt }}$ and $\tau_{\text {opt }}$. The operation current of the sensor is $100 \mu \mathrm{A}$.

The account of the error for setting the temperature in the course of a real experiment made it possible to determine the necessary and sufficient accuracy for location of the mesh points $T_{o p t}$ with mandatory condition (3). Our analysis of the obtained data has shown that the accuracy of setting the temperature should be: for $1 \mu \mathrm{A} \pm 0.3 \mathrm{~K}$ at temperatures below $30 \mathrm{~K}$, $\pm 3 \mathrm{~K}$ at temperatures above $30 \mathrm{~K}$, for $10 \mu \mathrm{A}- \pm 1.5 \mathrm{~K}$ over the entire operation range; for $100 \mu \mathrm{A}- \pm 0.3 \mathrm{~K}$ within the range of 30 to $80 \mathrm{~K}, \pm 1.5 \mathrm{~K}$ at temperatures above $80 \mathrm{~K}$. These precision are quite feasible for the installation of UGT-A.

\section{Conclusions}

1. It is shown that the developed SDTs are characterized by the five tolerance bands. The dispersion of sensor characteristics within a zone range from \pm 0.25 up to \pm $3.0 \mathrm{~K}$.

2. Developed is the algorithm for optimizing the calibration of interchangeable SDTs, which enables us to minimize the number of measurements needed to calculate thermometric characteristics with a given accuracy using the model of smoothing cubic splines with free knots. The proposed method makes it possible to significantly reduce time and material expenses associated with calibration of these sensors.

\section{References}

1. 1. P. Ciarlini, D. Ichim. Free-knot cubic spline modelling in cryogenic thermometer calibration // Measurement, 39(9), p. 815 - 820 (2006).

2. Yu. M. Shwarts, O. M. Ivashchenko, M. M. Shwarts. Metrological providing diode thermometry // Pribory, 8(86), p. 5 - 11 (2007).

3. O. M. Ivashchenko, Yu. M. Shwarts, M. M. Shwarts, D. P. Kopko, N.I. Sypko. Smoothing cubic spline approximation of thermometric characteristics for silicon diode temperature sensors // Semiconductor Physics, Quantum Electronics \& Optoelectronics, 14(4), p. ... (2010).

4. V. P. Denisyuk, B. G. Marchenko. Splines and their application to the tasks of modeling and processing the measuring signals. Kyiv, Published in Kyiv Polytechnical Institute, 1995. 246 p. 